\title{
Sífilis congénita temprana, serie de casos y descripción anatomopatológica
}

\section{Early congenital syphilis, case series and anatomopathological description}

\author{
Mónica Osorio-Guzmán,* Gabriel Santos-Vázquez, ${ }^{\ddagger}$, Daniel Torres-Muñoz, ${ }^{\S}$ \\ Graciela Inés González-Córdova," Erick Moreno-Pizarro, ${ }^{\ddagger \S}$ Diana Carolina Heredia-Cimental ${ }^{\S}$ \\ * Infectóloga Pediatra, Hospital Regional de León. \\ ₹ Fettes Promethea, A.C. \\ § Médico Interno de Pregrado, Universidad de Guanajuato, Departamento de Medicina y Nutrición. \\ " Jefa del Servicio de Neonatología, Hospital Ángeles León.
}

\begin{abstract}
RESUMEN
Introducción: La sífilis congénita es la infección sistémica provocada por Treponema pallidum adquirida por el neonato, vía transplacentaria de una madre que padece sífilis durante el embarazo. La infección fetal puede ocurrir en cualquier momento de la gestación. La transmisión vertical es más frecuente durante la sífilis secundaria. En México, la incidencia es de 2.9 casos por cada 100,000 recién nacidos. El cuadro clínico es diverso. Material y métodos: Siendo $50 \%$ mujeres y el resto hombres, la edad gestacional en promedio es de 38 semanas, la edad de diagnóstico de cinco casos fue en la primera semana de vida, en un caso hasta el cuarto mes. Respecto a las características maternas, la edad media fue 22 años, tres de ellas toxicómanas, cuatro no tuvieron control prenatal, las dos que lo tuvieron sólo acudieron a dos citas. Sólo una tuvo el diagnóstico de sífilis prenatal y sólo en ella se instauró tratamiento antenatal, pero de forma incompleta. Todos los pacientes tuvieron descamación y reactantes de fase aguda alterados, cinco tuvieron bajo peso al nacer y hepatoesplenomegalia. Neumonía alba, alteraciones óseas y en la biometría hemática se presentaron en dos ocasiones cada alteración. El VDRL (Venereal Disease Research Laboratory) fue positivo sólo en un paciente en líquido cefalorraquídeo. El VDRL sérico fue positivo en todos los casos con titulaciones desde 1:8 hasta 1:256, todos tuvieron serología negativa para VIH. En todos los casos el tratamiento fue penicilina $\mathrm{G}$ cristalina, en cuatro pacientes la dosis fue de $50 \mathrm{Ul} / \mathrm{kg} /$ dosis, y en dos de $300 \mathrm{Ul} / \mathrm{kg} /$ dosis, con duración de 10-14 días. Sólo un caso falleció, el resto vive. Conclusiones: La sífilis congénita es un problema de salud pública debido a la morbimortalidad para el neonato. En todos nuestros casos hubo un pobre o nulo control prenatal.
\end{abstract}

Palabras clave: Sífilis congénita temprana, Venereal Disease Research Laboratory, Treponema pallidum, neonato.

\section{ABSTRACT}

Introduction: Congenital syphilis is the systemic infection caused by Treponema pallidum acquired by the newborn, via the transplacental route of a mother suffering from syphilis during pregnancy. Fetal infection can occur at any time during pregnancy. Vertical transmission is most common during secondary syphilis. In Mexico the incidence is 2.9 cases per 100,000 newborns. The clinical picture is diverse. Material and methods: Being 50\% female and the rest male, the average gestational age at 38 weeks, age of diagnosis of 5 cases was in the first week of life, in one case up to the $4^{\text {th }}$ month. Regarding maternal characteristics, the average age was 22 years, 3 of them were drug addicts, 4 did not have prenatal control, the 2 who had it only attended 2 appointments. Only one was diagnosed with prenatal syphilis, and only one had antenatal treatment, but it was incomplete. All patients had desquamation and altered acute phase reactants, 5 had low birth weight and hepatosplenomegaly. Pneumonia Alba, bone alterations and in the hematic biometry were presented in 2 occasions each alteration. The VDRL (Venereal Disease Research Laboratory) was positive only in one patient in cerebrospinal fluid. Serum VDRL was positive in all cases with titres from 1:8 to 1:256, all were HIV negative. In all cases the treatment was crystalline penicillin $\mathrm{G}$ in 4 patients the dose of $50 \mathrm{lU} / \mathrm{kg} / \mathrm{dose}$, and in 2 of $300 \mathrm{IU} / \mathrm{kg} / \mathrm{dose}$, with duration of 10-14 days. Only one case died, the rest live. Conclusions: Congenital syphilis is a public health problem due to the morbidity and mortality for the newborn. In all our cases there was poor or no prenatal control.

Keywords: Early congenital syphilis, Venereal Disease Research Laboratory, Treponema pallidum, neonate.

Financiamiento: Ninguno.

Conflicto de intereses: Ninguno.

https://dx.doi.org/10.35366/95652 


\section{INTRODUCCIÓN}

La sífilis congénita (SC) es la infección sistémica provocada por Treponema pallidum adquirida por el neonato vía transplacentaria de una madre con sífilis durante el embarazo, así como durante el parto, en una madre con lesiones genitales. Un millón de mujeres embarazadas se infectan con sífilis a nivel mundial cada año. ${ }^{1}$ La SC conduce a 143,000 muertes perinatales de forma global. La tendencia mundial de la enfermedad ha ido en disminución, con una reducción del 39\% entre 2008 y 2012. Sin embargo, en los Estados Unidos de Norteamérica ha ocurrido lo contrario. ${ }^{2}$ En México de forma general se ha reducido el número de casos; sin embargo, la mayor prevalencia predomina en la región noroeste, en regiones aledañas a la frontera con Estados Unidos. ${ }^{3,4}$ En 2017 hubo 121 casos en el país, de los cuales el estado más afectado fue Baja California con 30 casos, seguido de Sonora con 20 casos. En Guanajuato se reportaron tres casos. ${ }^{5}$

La transmisión vertical ocurre en cualquier momento del embarazo, pero aumenta de acuerdo a la edad gestacional de forma proporcional. Asimismo, la sífilis secundaria es la que muestra mayor riesgo de transmisión vertical. ${ }^{6}$ En la actualidad, se sabe que la teoría respecto a la protección mediada por las células de Langerhans del citotrofoblasto no es efectiva para evitar la espiroquetemia desde el comienzo de la gestación. ${ }^{7} \mathrm{El}$ sistema inmunológico del neonato se caracteriza por inmadurez en sus barreras naturales, así como menor respuesta mediada por células NK, neutrófilos y una respuesta disminuida por parte de los linfocitos $T$ y $B$. Además de secretar una menor cantidad de citocinas y reactantes de fase aguda. ${ }^{8}$ Lo que lo lleva a ser proclive a condiciones infecciosas.

La sífilis congénita se divide en dos tipos, temprana y tardía, siendo el punto de corte la edad de dos años. Las manifestaciones clínicas de cada una de estas etapas pueden apreciarse en la Tabla 1.

Las pruebas serológicas útiles en el diagnóstico de la sífilis se pueden dividir en dos grupos:

a) Detección de anticuerpos heterólogos usando antígeno de origen no treponémico, USR (Unheated Serum Reagin), VDRL (Venereal Disease Research Laboratory), RPR (Rapid Plasma Reagin).

b) Detección de anticuerpos específicos usando antígenos de origen treponémico (FTA-ABS, TPHA), siendo FTA-ABS el estándar de oro para el diagnóstico de Sífilis. ${ }^{9}$

El objetivo de este trabajo es describir los casos de sífilis congénita temprana en un hospital de segundo nivel durante un periodo de dos años, así como la descripción específica de las características clínicas y

\begin{tabular}{|c|c|}
\hline $\begin{array}{l}\text { Sífilis congénita temprana } \\
\qquad(<2 \text { años })\end{array}$ & $\begin{array}{l}\text { Sífilis congénita tardía } \\
\qquad(>2 \text { años })\end{array}$ \\
\hline \multicolumn{2}{|c|}{ Examen físico } \\
\hline Óbito & Dientes de Hutchinson \\
\hline Prematuridad & Molares en mora \\
\hline Hidrops no inmune & Queratitis intersticial \\
\hline $\begin{array}{l}\text { Restricción de crecimiento } \\
\text { intrauterino }\end{array}$ & Sordera neurosensorial \\
\hline Hepatomegalia con o sin ictericia & Rágades \\
\hline Esplenomegalia & Retraso mental \\
\hline Rash cutáneo & Hidrocefalia \\
\hline $\begin{array}{l}\text { Adenopatía (suelen ser } \\
\text { epitrocleares) }\end{array}$ & Crisis convulsivas \\
\hline Rinitis & $\begin{array}{l}\text { Tabes dorsal, parálisis } \\
\text { nervios craneales }\end{array}$ \\
\hline Condiloma lata & Hueso frontal amplio \\
\hline Pseudoparálisis de Parrot & $\begin{array}{l}\text { Deformidad en silla de } \\
\text { montar de hueso nasal }\end{array}$ \\
\hline Coriorretinitis o cataratas & Paladar alto \\
\hline Crisis convulsivas & \\
\hline \multicolumn{2}{|c|}{ Radiografía } \\
\hline $\begin{array}{l}\text { Periostitis } \\
\text { Osteocondritis }\end{array}$ & $\begin{array}{l}\text { Engrosamiento } \\
\text { esternoclavicular (signo } \\
\text { de Higoumenákis), } \\
\text { articulación de Clutton }\end{array}$ \\
\hline Neumonía alba & Tibias de sable \\
\hline \multicolumn{2}{|c|}{ Otros } \\
\hline \multicolumn{2}{|c|}{$\begin{array}{l}\text { Síndrome nefrótico, pancreatitis, } \\
\text { miocarditis, diabetes insípida central }\end{array}$} \\
\hline \multicolumn{2}{|c|}{ Laboratorio } \\
\hline $\begin{array}{l}\text { Anemia } \\
\text { Trombocitopenia } \\
\text { Hipoglucemia } \\
\text { Pleocitosis o hiperproteinorraqu } \\
\text { Transaminasemia } \\
\text { Hiperbilirrubinemia directa }\end{array}$ & \\
\hline Elaboración propia. & \\
\hline
\end{tabular}


Tabla 2: Características clínicas de los pacientes.

\begin{tabular}{|c|c|c|c|c|c|c|}
\hline Paciente & 1 & 2 & 3 & 4 & 5 & 6 \\
\hline Sexo & Femenino & Femenino & Femenino & Masculino & Masculino & Masculino \\
\hline Edad gestacional al nacimiento (sdg) & 37 & 40 & 35 & 41 & 40 & 35 \\
\hline Edad al diagnóstico (días) & 5 & 2 & 1 & 0 & 120 & 1 \\
\hline LCR VDRL & ND & ND & ND & Positivo & Negativo & ND \\
\hline VDRL sérico & $1: 64$ & $1: 8$ & $1: 256$ & $1: 256$ & $1: 128$ & $1: 64$ \\
\hline Serología VIH ${ }^{\star *}$ & ND & ND & No reactivo & No reactivo & No reactivo & ND \\
\hline Hepatoesplenomegalia & $X$ & & $X$ & $x$ & $x$ & $X$ \\
\hline Bajo peso al nacer & $x$ & $x$ & $x$ & $x$ & & $x$ \\
\hline Pénfigo palmoplantar & $X$ & $x$ & $X$ & $x$ & $X$ & $X$ \\
\hline Fiebre & & & & & $x$ & \\
\hline Afección SNC & & & & $X$ & & \\
\hline Neumonía & & & & $x$ & $X$ & \\
\hline Datos radiográficos & & & & $x$ & $X$ & \\
\hline Leucocitosis & $x$ & $X$ & $X$ & $x$ & $X$ & $x$ \\
\hline Anemia & & & & & $X$ & $X$ \\
\hline Trombocitopenia & & & & $x$ & & $X$ \\
\hline PCR alterada & $x$ & $x$ & $X$ & $x$ & $X$ & $X$ \\
\hline Estado actual & Vivo & Vivo & Vivo & Vivo & Vivo & Muerto \\
\hline
\end{tabular}

Abreviaturas: sdg = semanas de gestación, LCR = líquido cefalorraquídeo, VDRL = Venereal Disease Research Laboratory, VIH = virus de inmunodeficiencia humana, $\mathrm{ND}=$ no disponible, $\mathrm{SNC}=$ sistema nervioso central.

anatomopatológicas de la enfermedad en el caso de un paciente con presentación grave y evolución letal.

\section{METODOLOGÍA}

Se realizó una revisión retrospectiva del periodo 01 de enero de 2016 a 31 de diciembre de 2018 de los archivos clínicos del Hospital General Regional de León Guanajuato de la SSA (Secretaría de Salud), se revisaron los expedientes de los pacientes con diagnóstico de sífilis congénita probable o confirmada. Se revisaron 18 expedientes, de los cuales se obtuvieron seis pacientes que cumplieron los criterios de inclusión (Tabla 2).

Se incluyeron todos los recién nacidos y lactantes con diagnóstico bioquímico de sífilis congénita que tuvieran expediente completo y disponible para su revisión. Se excluyeron aquéllos recién nacidos y lactantes que tuvieran sospecha del diagnóstico sin confirmación de éste, así como aquéllos cuyas madres tuvieron serologías positivas durante la gestación, pero el recién nacido fuera bioquímica y clínicamente negativo. Se reportan variables demográficas, clínicas y bioquímicas y de tratamiento tanto del neonato como de la madre.

\section{Definiciones operacionales ${ }^{9}$}

Caso confirmado. Se definió como niños y niñas con manifestaciones clínicas específicas de sífilis congénita con FTA-ABS IgM o se identificó Treponema pallidum por campo oscuro y/o IF de lesiones, cordón umbilical, placenta y necropsia, y con antecedentes maternos de VDRL y FTA-ABS y/o MHA-TP positivas.

Caso sospechoso: Se definió como niños y niñas con antecedente materno de VDRL o RPR y FTA-ABS y/o MHA-TP positivas.

Caso probable: Se definió como niños y niñas con manifestaciones clínicas específicas de sífilis congénita y con o sin antecedentes maternos de VDRL o RPR, FTA-ABS y/o MHA-TP positivas, que no hayan recibido tratamiento o hayan sido tratadas parcialmente en el embarazo.

Caso descartado: Se definió como niños y niñas con antecedente materno de VDRL y FTA-ABS y MHA-TP positivas pero sin manifestaciones clínicas específicas de sífilis congénita y con pruebas de laboratorio negativas.

\section{RESULTADOS}

De los recién nacidos 3/6 fueron mujeres, la edad gestacional en promedio fue de 38 semanas de 
gestación (35-41), la edad de diagnóstico de cinco casos fue en la primera semana de vida, en un caso hasta el cuarto mes. Sólo se pudo realizar VDRL en líquido cefalorraquídeo en dos pacientes, resultando positivo sólo en uno de ellos. EI VDRL sérico fue positivo en todos los pacientes, con titulaciones desde 1:8 hasta 1:256. Sólo se realizó serología para VIH en tres de seis pacientes, siendo negativo en los tres.

Respecto a los datos clínicos se presentó hepatoesplenomegalia en $5 / 6$ de los casos, bajo peso al nacer en 5/6, manifestaciones cutáneas como pénfigo sifilítico, alteraciones mucocutáneas y descamación palmoplantar en 6/6 de los casos. Fiebre en 1/6 de los casos, afección del sistema nervioso central en $1 / 6$, neumonía en $2 / 6$ de los casos. Alteraciones radiográficas como neumonía alba en $2 / 6$. El inicio de los síntomas hasta los cuatro meses en $1 / 6$, mientras que en el resto fue al primer día de vida, 5/6.
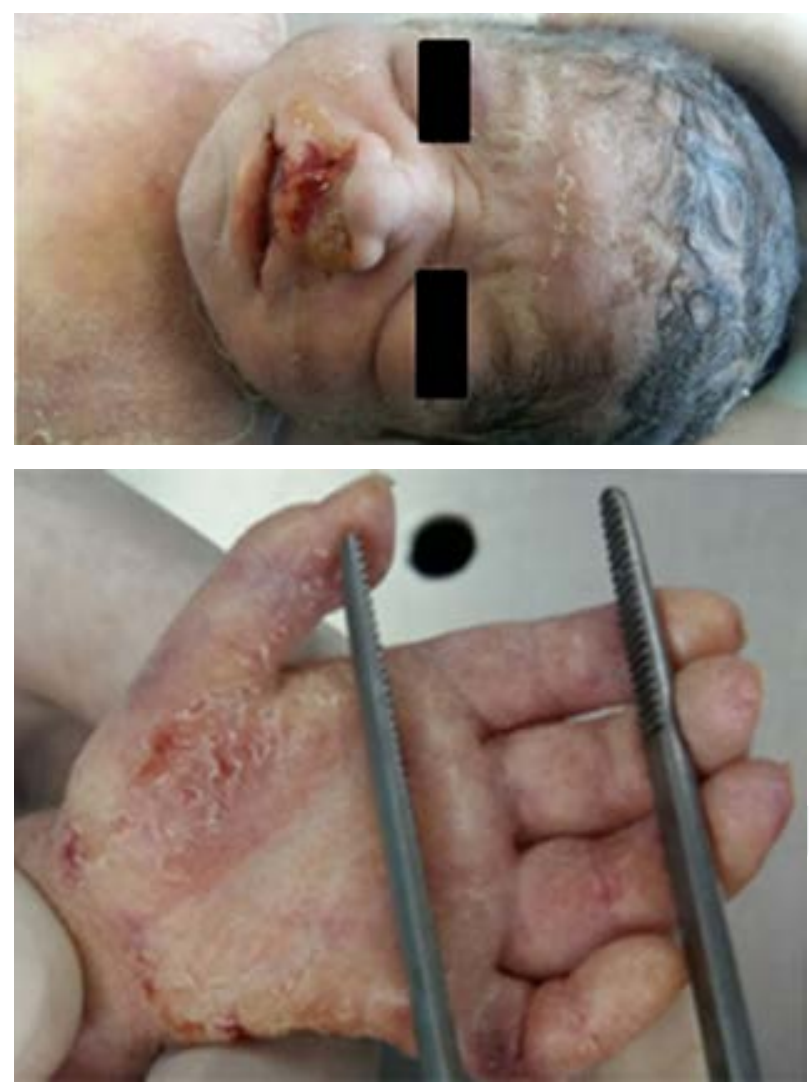

Figura 1: Paciente número 6 con manifestaciones típicas de la sífilis congénita temprana. Rinorrea serosanguinolenta. Descamación gruesa en región facial y palmar.

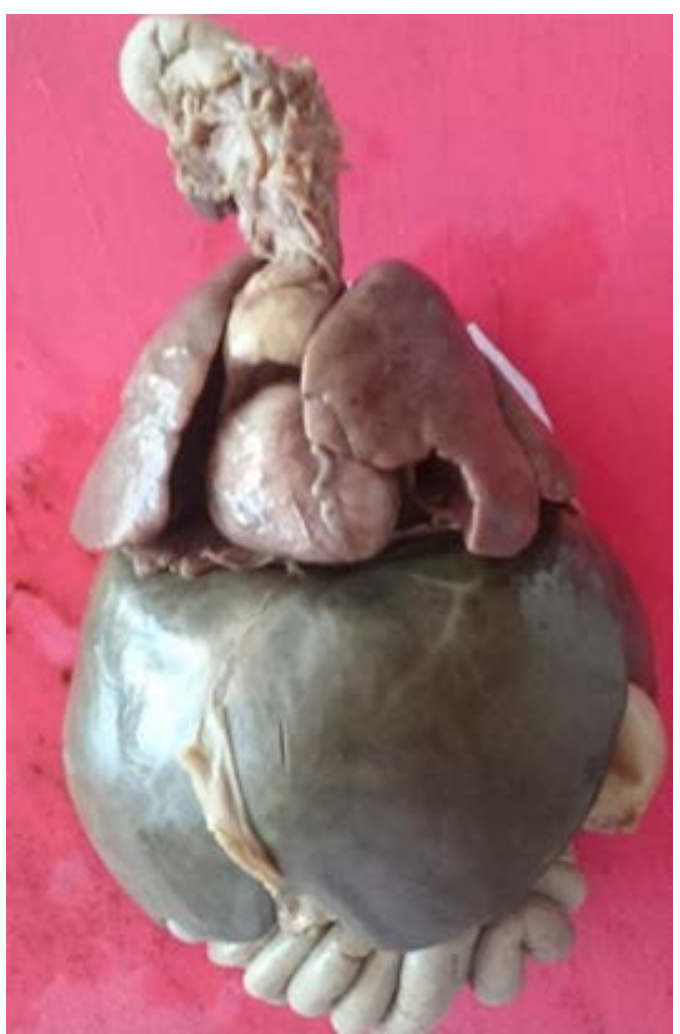

Figura 2: Hepatoesplenomegalia masiva, predomina la hepatomegalia, clínicamente palpable hasta la fosa iliaca derecha.

Las alteraciones por laboratorio fueron las siguientes: leucocitosis $6 / 6$ de los casos, anemia $2 / 6$, trombocitopenia $2 / 6$, proteína $C$ reactiva elevada $6 / 6$.

En el seguimiento, cinco pacientes sobrevivieron y completaron tratamiento específico, sólo uno falleció, en el cual se describen los hallazgos anatomopatológicos.

Respecto a las características maternas (Tabla 1), la edad promedio fue 22.3 años (19-29), presentaron toxicomanías $3 / 6$ de los casos, se desconoce la vía de éstas, solamente 2/6 tuvieron citas de control prenatal, siendo sólo dos citas de control en cada paciente, respectivamente. Se obtuvo VDRL en $2 / 6$, siendo reactivo en el $2 / 2$. Se obtuvo prueba rápida de VIH en 2/6 de los casos, siendo no reactivo en el 2/2. El diagnóstico de sífilis en las madres se realizó en forma postnatal en 5/6. Todas las madres recibieron tratamiento con penicilina, sólo una de ellas recibió tratamiento prenatal pero de forma incompleta, el resto comenzó el tratamiento postnatal. Todas las madres terminaron el esquema de tratamiento. 


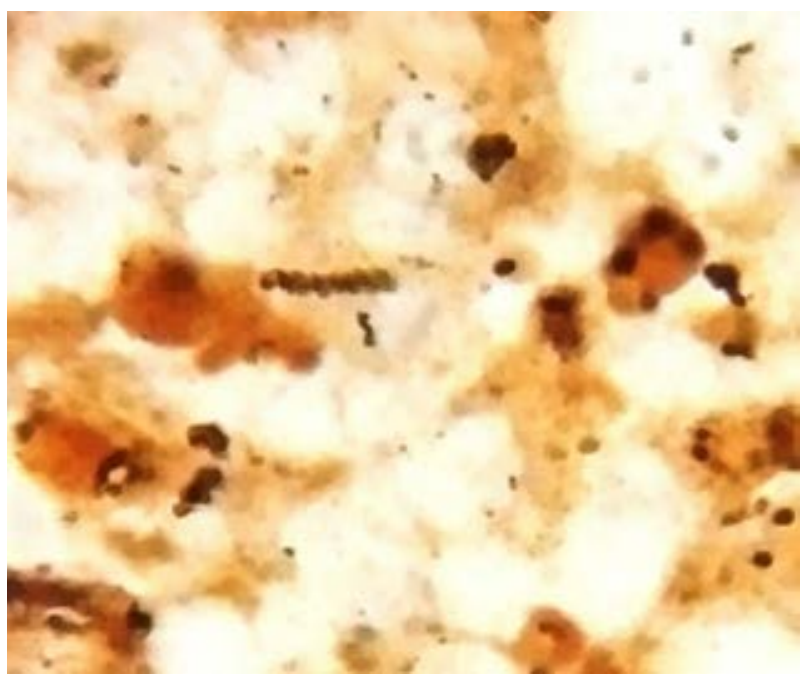

Figura 3: Tinción de Whartin-Starry en hígado, se aprecia una espiroqueta en el centro del campo, además de infiltrado difuso.

Se describe de forma particular el paciente número 6 , los datos clínicos y bioquímicos ya descritos. Se trata de paciente masculino de dos días de vida. Hijo de madre de 29 años sin control prenatal, asintomática. Acudió a las 36 semanas de gestación en trabajo de parto en fase activa y dilatación completa, con pérdida de bienestar fetal, obtenido vía vaginal. Teñido de meconio, no vigoroso, requirió un ciclo de ventilación con presión positiva e intubación orotraqueal, recuperó la frecuencia cardiaca; sin embargo, persistió esfuerzo respiratorio insuficiente. Apgar de 5/8. Se ingresó a UCIN. A la exploración se encontró en mal estado general, peso bajo para la edad gestacional, descamación gruesa en cara, tórax y extremidades (Figura 1), Silverman-Anderson 3 puntos, estertores basales bilaterales, abdomen globoso a expensas de hepatoesplenomegalia con límite inferior a $1 \mathrm{~cm}$ de espina iliaca. Se solicitó VDRL y se inició tratamiento con surfactante, aminas y penicilina. Su evolución fue tórpida y falleció a las 48 horas de vida debido a falla multiorgánica, se realizó autopsia del paciente, encontrándose marcada hepatoesplenomegalia (Figura 2). Se aplicó tinción de Whartin-Starry en pulmón, hígado y bazo. En hígado, se documentó hepatitis, necrosis, hematopoyesis extramedular y presencia de espiroquetas (Figura 3).

\section{DISCUSIÓN}

La sífilis congénita continúa siendo un problema de salud pública, a pesar de las medidas de prevención establecidas por la OMS y normadas también en nuestro país, no se ha logrado la erradicación de la enfermedad y, por tanto, su transmisión perinatal.

Como parte de la atención prenatal idónea, referida en las normas oficiales mexicanas, a todas las mujeres embarazadas se les debe realizar durante la primera visita prenatal, independientemente del trimestre de la gestación, la prueba de VDRL o RPR, útiles en la pesquisa inicial de sífilis, cuyo diagnóstico se confirma al resultar positivas las pruebas treponémicas FTA-ABS o al identificar la presencia del Treponema pallidum en biopsia del tejido o líquido sospechoso. ${ }^{10}$ Se debería considerar repetir la prueba de VDRL en los casos con factores de riesgo en diferentes momentos durante la gestación e inclusive previo al nacimiento. ${ }^{9}$

La prevalencia entre las series es distinta, pero alrededor de un $40-50 \%$ de los productos infectados por sífilis durante la gestación culminan en pérdida gestacional. ${ }^{6}$ El estudio de las mujeres con historia o en presencia de mortinatos o abortos espontáneos y nacimientos prematuros, con factores de riesgo para enfermedades de transmisión sexual, podría orientar y definir la causalidad de sífilis congénita y otras infecciones perinatales. ${ }^{11}$

La fisiopatología de la enfermedad supone una afección sistémica con infiltración por espiroquetas, alterando el flujo sanguíneo placentario, es la razón de las pérdidas gestacionales tempranas asociadas con la enfermedad. ${ }^{12}$

El riesgo de transmisión del agente de la madre hacia el feto es inversamente proporcional al tiempo de evolución del padecimiento en la madre, así como del tratamiento recibido por ésta. ${ }^{1}$

Los factores de riesgo para la presencia de SC son: embarazo sin control prenatal, falta de realización del diagnóstico de sífilis durante los primeros meses del embarazo, mujer con múltiples parejas sexuales o con una pareja sexual inestable, usuaria de drogas intravenosas o bien el tener relaciones con usuarios de drogas intravenosas, sexoservidora, haber cursado con el diagnóstico de sífilis en embarazo anterior y antecedente de otras enfermedades de transmisión sexual. ${ }^{9}$

Como se ha comentado, la SC se divide en temprana y tardía, la primera corresponde a la infección activa con inflamación, mientras que la tardía representa los estigmas y cicatrices acaecidos por la infección temprana. ${ }^{6}$

El diagnóstico de SC es complejo debido a que existe paso de anticuerpos IgG maternos (treponé- 
micos y no treponémicos) al feto, lo que dificulta la interpretación de los resultados serológicos. Hasta el momento no hay disponible una prueba diagnóstica específica que permita asegurar la presencia de infección en un recién nacido, pero sí existen elementos que orientan a éste. ${ }^{13}$

Los síntomas en el neonato con frecuencia son sutiles e inespecíficos y se estima que incluso el $60 \%$ de los niños afectados cursan asintomáticos al nacer, lo que hace que el diagnóstico dependa de los exámenes de laboratorio. Sin embargo, un grupo de estos pacientes suele ser etiquetados con diagnóstico de sepsis neonatal, sin llegar a un diagnóstico de certeza. El diagnóstico diferencial y la sospecha clínica es importante, ya que la instauración de tratamiento oportuno es clave para la resolución del padecimiento y disminuir morbimortalidad.

En nuestro trabajo se muestra la diversidad del espectro clínico de la enfermedad con tres casos clínicos de relevancia:

a) Neurosífilis congénita: se trata del paciente número 4, el cual presentó VDRL positivo en líquido cefalorraquídeo (LCR), las manifestaciones clínicas y bioquímicas fueron variadas y la respuesta al tratamiento adecuada. Se documentó negativización del VDRL en LCR y sérico tras el término del tratamiento.

b) Sífilis congénita de evolución fatal: se trata del paciente número 6 , del cual ya se han descrito sus características clínico-patológicas, se resalta la severidad de la infección y la afección multiorgánica característica de la enfermedad.

c) Sífilis congénita en lactante menor: se trata del paciente número 5 , el cual acude tras 120 días de nacimiento, debido a rinorrea persistente como signo pivote, con descamación palmar asociada. Multitratado sin mejoría. A su llegada a urgencias se inicia protocolo diagnóstico con los hallazgos ya comentados y tratamiento específico (Tabla 2).

La relevancia de estos casos clínicos radica en la diversidad clínica y su impacto en la morbimortalidad. Es importante no esperar hasta tener evidencia de las manifestaciones tardías de la sífilis congénita, como la tríada de Hutchinson u otras manifestaciones óseas características de la entidad. ${ }^{6}$ Siempre debe considerarse en los diagnósticos diferenciales de niños con probable sepsis neonatal, así como excluirse mediante tamizaje en las mujeres con factores de riesgo. ${ }^{9}$
Cabe señalar que de acuerdo con el número de casos reportados en este trabajo durante este periodo ( 3 años), el número de recién nacidos vivos fue de 21,855 . Por lo que se calculó la tasa de incidencia en este hospital, la cual fue de 0.27 por cada 1,000 nacidos vivos. Lo que sugiere un subregistro con los datos reportados a nivel local y nacional.

Es importante conocer la prevalencia de sífilis en adultos, pues mientras sea elevada, el riesgo de transmisión congénita es alto. ${ }^{11}$ Un grupo importante a considerar son los hombres jóvenes asintomáticos, los cuales fungen como reservorios para la enfermedad, en estados del norte de nuestro país, se ha documentado una mayor infección por esta espiroqueta en pacientes asintomáticos. ${ }^{14}$ Un grupo que actualmente cobra más relevancia son los migrantes, población con pobre o nula atención médica, expuesta a múltiples infecciones, entre ellas la sífilis, ${ }^{15}$ en nuestro país los movimientos sociales actuales podrían aumentar la prevalencia de esta infección; sin embargo, no se ha documentado hasta la fecha. El método más efectivo para reducir la sífilis congénita es disminuir la tasa de sífilis primaria, secundaria y latente en mujeres en edad reproductiva. ${ }^{16}$ Por lo cual se deben instaurar políticas públicas más efectivas para reducir esta infección.

\section{CONCLUSIONES}

La sífilis congénita es un problema latente asociado a embarazos no controlados como en nuestro medio y a infecciones tardías, mal tratadas o no tratadas. En nuestro país, se realizan estudios de pesquisa en las mujeres embarazadas; sin embargo, el control prenatal es deficiente, carece de oportunidad para el diagnóstico y para el tratamiento, pues menos de cinco consultas prenatales son consideradas como un control prenatal inadecuado, se concluye que no se realiza un escrutinio sistemático a las mujeres embarazadas, por lo que podría existir un subregistro de esta infección durante el embarazo.

Ningún recién nacido debe ser dado de alta, sin conocer la serología materna para sífilis, sobre todo en aquéllas que tienen factores de riesgo.

Existe la necesidad en nuestro medio de realizar actividades de promoción a la salud en la población objetivo, mujeres en edad fértil, adolescentes y usuarias de drogas principalmente intravenosas sobre infecciones de transmisión sexual y sexo seguro. 
Rev Latin Infect Pediatr 2020; 33 (3): 149-155

Se debe considerar el diagnóstico de SC temprana en todo neonato con sepsis y factores de riesgo independiente de la presentación clínica.

\section{REFERENCIAS}

1. Organization WH. Sexual and reproductive health. Eliminating congenital syphilis. WHO. 2017. [Accessed March 6, 2019] https://scholar.google.com.mx/ scholar?hl=es\&as_sdt=0\%2C5\&q=sexual+and+reproductiv e+health.+eliminating+congenital+syphilis\&btnG=.

2. Wijesooriya NS, Rochat RW, Kamb ML et al. Global burden of maternal and congenital syphilis in 2008 and 2012: a health systems modelling study. Lancet Glob Heal. 2012; 4 (8): e525-e533. doi: 10.1016/S2214-109X(16)30135-8.

3. Pineda-Leguízamo R, Villasis-Keever MÁ. Sífilis congénita: un problema vigente. Rev Mex Pediatr. 2017; 84 (2): 45-47.

4. California B. Prevalencia de sífilis congénita en tres hospitales públicos de Baja California, México, 2012-2015 Referencias Prevalencia de uso de antimicrobianos entre pacientes hospitalizados en áreas no críticas en un hospital universitario de México. 2017; 59 (5): 503-504.

5. SUIVE/EDGE/Secretaría de Salud/Estados Unidos Mexicanos 2017. Casos Nuevos de Sífilis Congénita (A50) Por Mes de Ocurrencia Estados Unidos Mexicanos 2017 Población General.; 2017. [Accesado 6 Marzo 2019] http://www. epidemiologia.salud.gob.mx/anuario/2017/casos/mes/092.pdf.

6. Porter S, Qureshi R, Children IB-I\& Y, 2018 undefined. Understanding Congenital Syphilis. journals.Iww.com. [Accessed 6 March 2019] https://journals.Iww.com/ iycjournal/Fulltext/2018/10000/Understanding_Congenital_ Syphilis.4.aspx.

7. Cooper JM, Sánchez PJ. Seminars in perinatology congenital syphilis. Semin Perinatol. 2018; 42 (3): 176-184. doi: 10.1053/j.semperi.2018.02.005.

8. Herrera A, Rodríguez T, Suárez A, Hernández B. El sistema inmune neonatal y su relación con la infección. Alergia, Asma e Inmunol Pediatr. 2013; 22 (3): 101-113.

9. Dirección General de Epidemiología. Manual de Procedimientos Estandarizados Para La Vigilancia
Epidemiológica de La Sífilis Congénita. México: Secretaría de Salud; 2012.

10. Herrera-Ortiz A, López-Gatell H, García-Cisneros S et al. Sífilis congénita en México. Análisis de las normas nacionales e internacionales desde la perspectiva del diagnóstico de laboratorio. Gac Med Mex. 2019; 155 (5): 464-472.

11. Álvarez HG, Salazar AS, Bocanegra LC. Guía diagnóstico y manejo de la sífilis en el embarazo y prevención de la sífilis congénita. Sonora, México: Secretaría de Salud Pública del Estado de Sonora; 2012. [Accesado 15 Diciembre 2019] http://www.censida.salud.gob.mx/ descargas/normatividad/guia_diagnostico_manejo_sifilis_ congenita.pdf.

12. Benenson I, Cen F. Understanding Congenital Syphilis. 2018; 31 (4): 287-296. doi: 10.1097/IYC.0000000000000125.

13. Álvarez CR. Interpretación de las pruebas diagnósticas de sífilis en gestantes. Rev Peru Ginecol y Obstet. 2018; 64 (3): 345-352. doi: 10.31403/rpgo.v64i2095.

14. Herrera-Ortiz A, Uribe-Salas FJ, Olamendi-Portugal ML, García-Cisneros S, Conde-Glez CJ, SánchezAlemán MA. Análisis de la tendencia de sífilis adquirida en México durante el periodo 2003-2013. Salud Pública Méx. 2015; 57 (4): 335-342. [Accesado 15 Diciembre 2019] https://www.medigraphic.com/cgi-bin/new/resumen. cgi?IDARTICULO=61126.

15. Kousoulis AA, Stavrianeas N, Karamanou M, Androutsos G. Social aspects of syphilis based on the history of its terminology. Indian J Dermatol Venereol Leprol. 2011; 77 (3): 389-391. doi:10.4103/0378-6323.79734.

16. Pérez KV, Cedillos CM, Bahena MT, Valencia A. Sífilis congénita, la gran simuladora. Dermatología Rev Mex. 2014; 58 (1): 40-47. [Accesado 5 Diciembre 2019] https://www.medigraphic.com/cgi-bin/new/resumen. cgi?IDARTICULO=48547.

Correspondencia:

Gabriel Santos Vázquez

E-mail: gabosantos23@gmail.com 